matische Systeme anwendbar sein kann. Wir hatten diese Tatsache auch im Titel unserer Abhandlung zum Ausdruck gebracht.

In seiner Notiz ${ }^{6}$ über Singulett- und Triplettzustände einfacher Kohlenwasserstoffe stellt Seel fest, daß das von ihm für Trimethylenmethyl berechnete Termschema nicht mit dem ven uns früher ${ }^{7}$ publizierten übereinstimmt. Tat- sächlich ist die Abbildung unserer Notiz fehlerhaft, und wir haben vor dem Erscheinen der Seelschen Notiz in dieser Zeitschrift eine Berichtigung ${ }^{8}$ gebracht. Die Seelschen Rechnungen bestätigten also unsere Resultate.

6 F. S e e l, Z. Naturforschg. 3a, 180 [1948].

7 H. H a r t m a n n, Z. Naturforschg. 2a, 684 [1947].

- H. H a r tm a n n, Z. Naturforschg. 3a, 128 [1948].

\title{
Die Temperaturabhängigkeit des Stoßfaktors in einer Formel für die Schallgeschwindigkeit in Flüssigkeiten
}

\author{
Von Daniele Sette \\ Istituto Nazionale di Ultracustica „O. M. Corbino“, Roma \\ (Z. Naturforschg. 5 a, 170-171 [1950]; eingegangen am 21. Januar 1950)
}

\begin{abstract}
An Hand der Ausdrücke für die Fortpflanzungsgeschwindigkeit von Ultraschall von W. S cha a f $\mathrm{s}$ und M. R a o wird für nicht assoziierte Flüssigkeiten der Stoßfaktor als Funktion des Verhältnisses $T / T_{\mathrm{k}}\left(T_{\mathrm{k}}=\right.$ kritische Temperatur $)$ berechnet. Die experimentellen Ergebnisse stehen mit der Rechnung im Einklang.
\end{abstract}

1. W. S cha aff $\mathrm{s}^{1}$ hat kürzlich in einer Arbeit über den Zusammenhang zwischen Schallgeschwindigkeit $v$ und Molekülstruktur folgende halbempirische Formel aufgestellt, die für organische Flüssigkeiten gilt:

$$
v=w\rceil R T s r .
$$

Darin ist $w$ eine Konstante der Dimension $M^{-1 / 2}$, $r$ ist die Raumerfüllung der Moleküle und $s$ der Stoßfaktor, der bei der Annahme völlig elastischer Stöße zwischen den Molekülen nach van der Waals den Wert 4 hat, in Wirklichkeit aber kleiner ist.

Die Raumerfüllung $r$ ist das Verhältnis zwischen dem effektiven Molekülvolumen pro Mol $B$ und der Molvolumen $V$.

Das vom Molekül wirklich eingenommene Volumen kann in erster Näherung als von der Temperatur unabhängig angesehen werden. Es steht im Zusammenhang mit dem Covolumen durch die Beziehung $b=s_{\mathrm{n}} B$, wobei der Proportionalitätsfaktor 'von der Temperatur abhängt, da bekanntlich auch $b$ eine Funktion der Temperatur ist.

Der Stoßfaktor ist nach Schaaffs durch die Beziehung definiert:

$$
s=s_{\mathrm{n}}\left(1-r_{\mathrm{o}} / r\right) .
$$

Die innermolekulare Größe $r_{0}$ wurde zu dem Zweck eingeführt, um (1) auch in Spezialfällen mit den Versuchen in Übereinstimmung zu bringen. Es ist jedoch zu bemerken, daß sie nur dann einen merklichen Wert

1 W. S ch a aff s, Z. Naturforschg. 3a, 396 [1948].

2 M. R a o, Indian J. Physics Proc. Indian Assoc. Cultivat. Sci. 14, 109 [1940]. besitzt, wenn das Molekül schwere Atome (wie Halogene) oder spezielle Konfigurationen enthält.

In den Überlegungen von Schaaffs kommt dem Stoßfaktor eine bemerkenswerte Bedeutung zu, und wir erachten es als nützlich, seine Abhängigkeit von der Temperatur zu prüfen. Dies ist möglich durch Vergleich mit der empirischen Beziehung von M. Rao, welche die Temperaturabhängigkeit von $v$ anzugeben gestattet.

2. M. R. R a o ${ }^{2}$ hat empirisch die Gültigkeit folgender Beziehung für nicht assoziierte organische Flüssigkeiten nachgewiesen:

$$
v^{1 / 3} V=\Re,
$$

worin $\Re$ von der Temperatur unabhängig ist.

Die gleichzeitige Gültigkeit von (1) und (3) und die Annahme ihrer Unabhängigkeit von einander erlaubt es, bei solchen Flüssigkeiten für $s$ zu erhalten:

$$
s=\frac{\mathfrak{R}^{3}}{w / R T M^{2} B \frac{\varrho^{2}}{\sqrt{T}},}
$$

wobei $M$ das Molekulargewicht und $\varrho$ die Dichte sind.

Für nicht assoziierte Flüssigkeiten kann außerdem die Dichte im Bereich zwischen Schmelzpunkt $\left(T_{\text {Schm }_{1}}\right)$ und kritischer Temperatur $\left(T_{\mathrm{k}}\right)$ ausgedrückt werden durch:

$$
\varrho-\bar{Q}=\varrho_{0}\left(1-T / T_{\mathrm{k}}\right)^{0,3},
$$

wo $\bar{\varrho}$ die Dampfdichte bei der Temperatur $T$ ist $^{3}$.

3 S. S u d g e n, J. Chem. Soc. [London] 1927, 1780; 1929,1055 . 


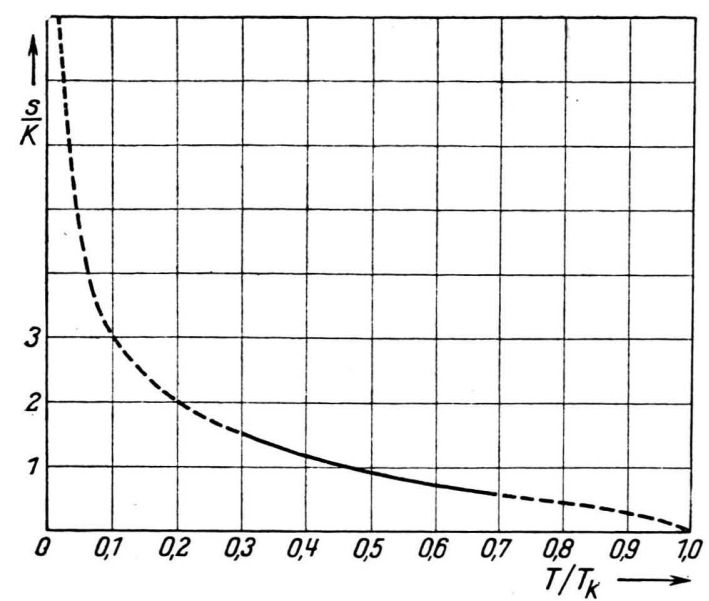

Abb. 1. $s / K$ als Funktion von $T / T_{\mathrm{k}}$.

Solange man sich nicht sehr nahe bei der kritischen Temperatur befindet, und das ist, wie man gleich sehen wird, der uns interssierende Fall, kann $\bar{o}$ gegenüber $\varrho$ vernachlässigt werden. Aus (4) und (5) ergibt sich dann mit

$$
\begin{gathered}
K=\frac{\Re^{3} \underline{O}_{0}{ }^{2}}{w \sqrt{R T_{\mathrm{k}} M^{2} B}:} \\
s=K\left(1-\frac{T}{T_{\mathrm{k}}}\right)^{0,6} \frac{1}{\sqrt{T / T_{\mathrm{k}}}},
\end{gathered}
$$

wo der Koeffizient $K$ von verschiedenen, die Flüssigkeit charakterisierenden Größen abhängt, aber von der Temperatur unabhängig ist.

3. Abb. 1 stellt das Verhältnis $s / K$ als Funktion von $T / T_{\mathrm{k}}$ dar. In Tab. 1 sind für eine Reihe von Flüssigkeiten die kritische Temperatur $T_{\mathrm{k}}$ sowie die Verhältnisse $T_{\mathrm{schm}} / T_{\mathrm{k}}, 293,1 / T_{\mathrm{k}}$ und $T_{\mathrm{s}} / T_{\mathrm{k}}$ zusammengestellt. Die Daten entstammen den Landolt-Börnstein-Tabellen und den International Critical Tables. Das Verhältnis $T / T_{\mathrm{k}}$ nimmt bei Flüssigkeiten im allgemeinen Werte zwischen 0,3 und 0,65 ein, und dies ist folglich dasjenige Gebiet aus Abb. 1, welches uns interessiert. Die Funktion ist darin nahezu linear.

Es ist danach verständlich, daß die prozentuale Geschwindigkeitsänderung in einem bestimmten Tem-

\begin{tabular}{|c|c|c|c|c|}
\hline Substanz & $T_{\mathrm{k}}$ & $T_{\mathrm{Schm}}$ & 293,1 & $T_{\mathrm{s}}$ \\
& & $T_{\mathrm{k}}$ & $T_{\mathrm{k}}$ & $T_{\mathrm{k}}$ \\
\hline & & & & \\
Aceton . . . . . . . & 507,5 & 0,350 & 0,577 & 0,649 \\
Acetonitril . . . . . & 547,8 & 0,423 & 0,535 & 0,648 \\
Athylacetat . . . . . & 523,2 & 0,362 & 0,560 & 0,669 \\
Äther . . . . . . & 466,3 & 0,336 & 0,628 & 0,659 \\
Äthylalkohol . . . . . & 516,2 & 0,301 & 0,567 & 0,681 \\
Benzol . . . . . . . & 561,6 & 0,496 & 0,522 & 0,628 \\
Chlorbenzol . . . . . & 632,3 & 0,360 & 0,463 & 0,640 \\
Chloroform . . . . . & 536,1 & 0,390 & 0,546 & 0,623 \\
Cyclohexan . . . . . & 554,1 & 0,504 & 0,528 & 0,639 \\
Heptan . . . . . . & 539,95 & 0,338 & 0,542 & 0,688 \\
$n$-Hexan . . . . . . & 507,9 & 0,352 & 0,577 & 0,673 \\
Methylalkohol . . . & 516,1 & 0,341 & 0,571 & 0,658 \\
$n$-Pentan . . . . . & 470,3 & 0,301 & 0,623 & 0,657 \\
$n$-Propylalkohol . . . & 536,8 & 0,272 & 0,546 & 0,689 \\
$n$-Octan . . . . . . & 569,1 & 0,380 & 0,515 & 0,700 \\
Schwefelkohlenstoff . & 546,1 & 0,301 & 0,536 & 0,584 \\
Tetrachlorkohlenstoff & 556,25 & 0,450 & 0,527 & 0,627 \\
Toluol . . . . . . . & 593,7 & 0,300 & 0,493 & 0,647 \\
Xylol . . . . . . . . & 631,4 & 0,386 & 0,464 & 0,660 \\
& & & & \\
\hline
\end{tabular}

Tab. 1.

peraturbereich (z. B. $0 \ldots 50^{\circ} \mathrm{C}$ ) für verschiedene Substanzen ungefähr gleich groß ist, wenn ihre kritischen Temperaturen nicht sehr voneinander abweichen.

Dieser Umstand erklärt die Beobachtungen von Schaaffs beim Vergleich der empirischen Werte der Geschwindigkeiten für verschiedene Substanzen bei 0 und $50^{\circ} \mathrm{C}$. Er fand tatsächlich für verschiedene, nicht assoziierte Flüssigkeiten nahezu gleiche Variationen von $s(21 \%$ bis $23 \%)$.

Die experimentellen Ergebnisse stehen mit der Rechnung in Einklang. Schaaffs, z. B., findet experimentell zwischen 0 und $50^{\circ} \mathrm{C}$ für $s$ einen Unterschied von $21,8 \%$ in Aceton, $21,4 \%$ in Chloroform und $21,4 \%$ in Toluol, während die aus (7) zu erwartenden Werte in der gleichen Reihenfolge 25\%, 23\% und $21 \%$ sein sollten.

Da $\frac{1}{s} \frac{d s}{d T}$ von strukturellen Elementen unabhängig ist, bestätigt sich die Meinung von Schaaffs, wonach die Temperaturabhängigkeit von $s$ in (2) im wesentlichen auf $s_{\mathrm{n}}$ zurückzuführen ist.

Der Verfasser dankt Hrn. Dr. W. S c ha aff s vielmals für sein gütiges Interesse an der vorliegenden Arbeit. 